\title{
El potencial del podcast como recurso didáctico para el desarrollo de las destrezas orales de segundas lenguas con dispositivos móviles
}

\author{
RAÚl SANTIAGO \\ Universidad de La Rioja \\ ELENA BÁRCENA \\ Universidad Nacional de Educación a Distancia, UNED
}

Recibido: 5 marzo 2016 / Aceptado: 18 abril 2016

ISSN: 1697-7467

\begin{abstract}
RESUMEN: Este artículo presenta los podcasts y el podcasting para la distribución de contenidos de cualquier índole para que puedan escucharse o verse tanto en un equipo informático o, mejor, en un dispositivo móvil, como las tabletas y los smartphones. Se analiza la aplicación de los podcasts al aprendizaje de segundas lenguas y en particular la adecuación de su uso en el desarrollo de destrezas orales, tanto receptivas (comprensión) como productivas (habla) y, dentro de estas últimas, a aspectos como la pronunciación, la entonación y la fluidez. Aquí se señalan los diferentes tipos de podcasts que cabe distinguir, así como las ventajas y logros alcanzados hasta la fecha en una muestra ilustrativa de proyectos de investigación y experiencias didácticas. Finalmente, se reflexiona sobre la necesidad de integrar los podcasts con otras propuestas innovadoras metodológicas y tecnológicas que están surgiendo en paralelo para dar respuesta a los retos educativos del siglo XXI en materia de segundas lenguas.
\end{abstract}

Palabras clave: Podcasts, dispositivos móviles, aprendizaje de segundas lenguas, competencias orales.

The Potential of Podcasts as a Didactic Resource for the Development of Oral Skills in Second Language with Mobile Devices

\begin{abstract}
This article presents podcasts and podcasting for the distribution of contents of any kind that can be listened to or watched on a computing equipment or, better, on a mobile device, such as a tablet or a smartphone1. The application of podcasts to the learning process of second languages, and particularly, the adequacy of its use for the development of oral skills is analyzed, including both receptive skills (comprehension) and productive skills (speaking) and, within the latter, aspects such as pronunciation, intonation, and fluency. Here, a fundamental distinction is made according to the type of podcasts, and the affordances and achievements that have been found until now in research projects and didactic experiences. Finally, a reflection is made about the need to integrate podcasts with other innovative methodological and technological proposals that are emerging in parallel, in order to provide a solution to the several educational challenges regarding second languages in the 21 st Century.
\end{abstract}

Keywords: Podcasts, mobile devices, second language learning, oral competences.

\footnotetext{
${ }^{1}$ Parte de la investigación en la que se enmarca este artículo ha sido financiada por el Ministerio de Economía y Competitividad en el proyecto de investigación SO-CALL-ME (FFI-2011/29829).
} 


\section{INTRODUCCIÓN}

En la sociedad globalizada, móvil e hiperconectada en la que vivimos, las necesidades de formación e información se han multiplicado y, consecuentemente, han proliferado los recursos tecnológicos para cubrir una y otra. Se observa también que las propuestas proceden de sectores heterogéneos, con los consiguientes cambios de perspectivas y roles. Un ejemplo de dicha tecnología, que ha llegado al mundo de la educación procedente del de la información y los medios de comunicación son los podcasts. Se trata de emisiones de radio o de televisión que un usuario puede descargar de internet, mediante un sistema de redifusión (conocido como RSS [Rich Site Summary]) y una suscripción previa, para acceder a ellas desde un ordenador o dispositivo digital. Se trata, por lo tanto, eminentemente de audio y video, si bien pueden ir acompañados de notas o subtítulos, que los hacen más informativos y accesibles. Las ventajas de este formato han sido apreciadas desde múltiples perspectivas, desde la de los propios comunicadores, que han visto su audiencia crecer exponencialmente, hasta la del público, por la flexibilidad que conlleva acceder a los contenidos cuando se desee.

Los podcasts constituyen una creación del presente siglo, si bien antes ya existían programas radiofónicos que colgaban sus contenidos en Internet. Sin embargo, fue realmente con la invención del RSS cuando apareció el concepto de podcasting y comenzó a evolucionar en campos cada vez menos próximos a la información general sobre la actualidad tal, y como se entiende de forma convencional. Dichos campos incluyen, por ejemplo, la divulgación artística y científica, el turismo (p.ej., visitas guiadas) y la formación. En cuanto a este ultimo, se observa que desde el comienzo de la primera década del siglo XXI se ha venido generando un considerable volumen de estudio por parte de la comunidad docente y investigadora (Veiga Marriott \& Lupion Torres, 2009). Sitios web como los de la BBC, Newsweek y podcastellano.com ofrecen podcasts para ser descargados y oídos. Esto ha conllevado la creación de comunidades virtuales concebidas como almacén y el desarrollo de herramientas que permiten acceder a estos contenidos desde cualquier dispositivo electrónico.

La llegada del siglo XXI también ha sido testigo del crecimiento exponencial del uso de los dispositivos móviles, que nacieron en el ámbito de las telecomunicaciones, pero cuyas múltiples ventajas de flexibilidad de uso por su portabilidad, uso, etc. y su creciente sofisticación que las ha convertido en verdaderas 'navajas suizas' (Read \& Bárcena, 2015), han hecho que su uso haya sido diversificado en otros ámbitos de la realidad social como la educación. Años después de las primeras apps y los primeros estudios para aprovechar las funcionalidades de los móviles con fines didácticos, esta tecnología continua influyendo en la forma en la que las segundas lenguas pueden ser practicadas y estudiadas. Son muchos los autores que han constatado como los teléfonos móviles y las tabletas ofrecen una forma altamente adecuada para integrar la tecnología digital en el proceso de aprendizaje de segundas lenguas. El enorme avance en la posesión de estos dispositivos entre la población junto con el acceso fiable y robusto a contenidos que se pueden encontrar en línea implica ha estimulado tanto la oferta como la demanda de aplicaciones formativas, que incluyen las segundas lenguas. En 2015, con aproximadamente millón y medio de apps disponibles para descarga en tiendas de apps como Google Play and iTunes, instituciones, instructores y estudiantes tienen la oportunidad de aplicar las formas innovadoras de acceso y manejo de contenidos útiles para la adquisición de conocimientos y desarrollo de competencias y destrezas enormemente diversas y la comunidad investigadora no ha cesado en su empeño 
de seguir ideando nuevas a medida que avanzan los modelos pedagógicos y progresan las capacidades tecnológicas, en una especie de espiral que se retroalimenta continuamente. En los siguientes apartados profundizaremos en el concepto de podcast y analizaremos su potencial didáctico y, en particular, en el contexto de las segundas lenguas en formato móvil.

\section{Concepto y antecedentes del PODCAST}

Como se anticipaba en la Introducción, el podcast es un medio de distribución de contenidos de cualquier índole para que puedan escucharse o verse tanto en el ordenador como en un reproductor portátil o un teléfono móvil. Técnicamente, el podcast consiste en crear archivos de sonido (generalmente en mp3 u ogg) y poder subscribirse mediante un archivo RSS de manera que permita que un programa lo descargue para que el usuario lo escuche en el momento que quiera, generalmente en un reproductor móvil. Si bien el término podcasting se acuñó a principios del 2004 (combinando los términos iPod y broadcasting), el concepto fue demostrado tres años antes por Dave Winer (el padre del podcasting y del RSS) cuando incluyó un audioblog con una canción de Grateful Dead en un enclosure tag de RSS (lo que se conoce hoy como un podcast) en RadioUserland, la primera herramienta para construir blogs (inventada también por Winer).

Trabajando con Winer, Adam Curry publicó su primer podcast en octubre del 2002 en RadioUserland. El problema en esos años era que el único agregador que leía enclosure tags era RadioUserland y, por lo tanto, la posibilidad de difusión de un podcast era muy limitada. En octubre del 2003, se pusieron a disposición del público una serie de programas de Chris Lydon de la Radio Pública Nacional para ser distribuidos como podcasts y fue en en esa misma época cuando comenzaron a aparecer los primeros agregadores de RSS para uso general capaces de leer el enclosure tag (Amphetadesk fue el primero: http://rasterweb.net/ raster/ 2003/09/24/20030924083605/). Simultáneamente (en octubre 2003), durante la primera conferencia BloggerCon, Adam Curry presentó su primer Applescript que descargaba mp3s al iPod automáticamente (http://arstechnica.com/business/2014/08/10-years-of-podcasting-codecomedy-and-patent-lawsuits/). Durante su demostración Curry animó encarecidamente a la audiencia a desarrollar scripts y agregadores para uso más general y a difundir el fenómeno.

Posteriormente a BloggerCon 2003, comenzó el trabajo con iPodderX (para Mac OS X) y se vio la necesidad de darle al fenómeno un nombre diferente a audioblogging, así que a principios del 2004 se acuñó el término podcasting para diferenciar este nuevo concepto del de audioblogging. Con la salida del iPodder para otras plataformas en septiembre de 2004, se inició el verdadero despegue del podcasting. Muchos acuden a esa fecha, septiembre de 2004, para referirse al inicio del fenómeno pero, siguiendo este análisis, parece más razonable establecer que el podcasting en realidad se creó o inició en enero del 2001 y que se popularizó o empezó su difusión masiva en septiembre del 2004. El término podcasting se utilizó por primera vez el 12 de febrero de 2004 en el periódico The Guardian, aunque no hacía referencia a la sincronización automática. Fue Hammersley quien lo hizo en un artículo titulado 'Audible Revolution', publicado en la edición digital de The Guardian en febrero de 2004, quien hablaba en su reportaje de una "revolución del audio amateur" (http://www. theguardian.com/media/2004/feb/12/broadcasting.digitalmedia). Es a partir de esta fecha en 
que el concepto queda ya definido y comienzan a registrarse los dominios relacionados con el término como podcasting.net o podcasting.es y a partir de 2010, han ido apareciendo diversos programas que han facilitado la creación y la distribución de podcasts por Internet.

En cuanto a los usos relacionados con los podcasts, la red Guadalinfo (http://es-hn. recuweb.com/guadalinfo) destaca una larga lista, que incluye los siguientes:

- Televisión y radio: la mayoría de programas de radio emiten la totalidad de sus contenidos y cada vez más programas de televisión emiten en su canal de podcasting sus partes más influyentes en la audiencia.

- Entrevistas: posibilidad de recibir las entrevistas completas en el podcasting del programa.

- Congresos: posibilidad de transmitir mediante el podcasting las comunicaciones, ponencias y mesas redondas a los asistentes.

- Formación abierta y a distancia: posibilidad de estudiar en cualquier momento y en cualquier lugar.

- Aplicaciones educativas: los alumnos pueden recibir las lecciones lectivas que deseen, así como estudiar cuando el alumnado lo crea conveniente.

Es evidente que dada esta diversidad de aplicabilidad, era cuestión de tiempo que surgieran las primeras exploraciones de los podcasts en el contexto del aprendizaje de segundas lenguas. Analizar el potencial de los podcasts para esta finalidad en concreto es el objetivo del siguiente apartado.

\section{El podcast en el aprendizaje de UNA SEgunda lengua}

La adecuación de los podcasts para el aprendizaje de una segunda lengua es evidente cuando se ve como está de extendido como recurso didáctico en este ámbito y en un gran número de sitios web (como Mosalingua ${ }^{2}$ y Examtime ${ }^{3}$, por nombrar algunos) se puede localizar una gran cantidad de estos podcasts. El abanico es muy amplio: desde el empleo de canales genéricos de noticias internacionales de política y economía hasta la utilización de recursos específicamente diseñados para la ejercitación y mejora de determinados aspectos lingüísticos como la fonética, el vocabulario o las peculiaridades de los dominios lingüísticos especializados o lenguas para fines específicos. También los documentales sobre ciencia, tecnología e historia pueden ser muy útiles en las manos de un profesor en el aula de lenguas que puede poner en marcha iniciativas metodológicas como el aprendizaje basado en proyectos o el aprendizaje colaborativo y emplear podcasts con sintéticos monográficos especializados que se pueden asignar entre los estudiantes con el fin de elaborar un proyecto común más amplio.

El podcast puede incluir una serie de contenidos de sonido o visuales que se han de analizar y trabajar o puede ser el propio estudiante quien revise, reenfoque o integre los contenidos o incluso llegue a crear los suyos propios, siguiendo enfoques ampliamente aceptados en la comunidad académico-investigadora como son el aprendizaje autónomo y

\footnotetext{
${ }^{2}$ http://www.mosalingua.com/es/los-mejores-podcast-para-aprender-ingles/

${ }^{3} \mathrm{https}: / / \mathrm{www}$. examtime.com/es/blog/mejores-podcasts-educativos/
} 
personalizado. Llevar a cabo estos procesos le conducirá a tener que realizar un esfuerzo de asimilación y optimización para crear un producto coherente y eficaz, por no mencionar los beneficios del desarrollo de la competencia digital, que por su transversalidad seguramente encontrará de su utilidad en otras facetas de su vida personal, social o profesional.

En cuanto a la integración del podcasting en el aula de segundas lenguas, está claro que no pretende, ni debe hacerlo, sustituir al profesor, sino que se trata de otro instrumento más a disposición del profesor y estudiante para reforzar el aprendizaje y lograr otros nuevos. Como elemento tecnológico que es, las ventajas del podcasting - y más aún es soporte móvil - son similares a las de tantas otras como los audioblogs que han sido analizadas en la literatura (López Vidales \& González Aldea, 2014), tales como el hecho de que se libera el aprendizaje de limitaciones espaciotemporales. Ello conlleva, como también es el caso de otras tecnologías, un replanteamiento del rol del profesor y de la responsabilidad y protagonismo primordiales que debe ejercer el estudiante en su propio proceso de aprendizaje.

Los reparos más comunes hacia el uso de recursos didáctico-tecnológicos como los podcasts incluyen el temor a que tanta disponibilidad puede conducir a una disminución de la presencialidad de los estudiantes en los centros educativos (ésta parece ser una de las paradojas del uso de la tecnología). Si bien cabe la posibilidad de que un estudiante altamente motivado, autorregulado, etc. llegue a configurar un diseño personalizado de forma completamente autónoma, la larga trayectoria del aprendizaje asistido por ordenador demuestra que en la inmensa mayoría de los casos, estas herramientas se traducen didácticamente en una complementariedad a la sesión presencial o incluso algún tipo de trabajo previo, tal y como como postulan los defensores del modelo flipped classroom (Tourón, Santiago \& Díez, 2014). En cualquier caso, las experiencias de aprendizaje con podcast hasta la fecha no parecen evidenciar que se haya producido una disminución de la presencialidad:

[...] Assist auditory learners. Proponents of podcasting point out that the medium is perfect for learners who prefer to take in information aurally. Margaret Maag, an assistant professor at the University of San Francisco's School of Nursing, has recorded her classroom lectures and posted them on a secure website since learning about podcasting from an Educause webinar in March 2005. She explains to students that the purpose is to help audio learners retain the information covered. Even though critics initially said students would stop attending classes, Maag found that attendance did not in fact decline, because students "didn't want to miss what was going on".

De aquí se deduce que el éxito o impacto del uso de los podcasts en el aprendizaje de lenguas depende tanto del diseño de la propia actividad que los emplee, como de las anteriores y las posteriores, es decir, del diseño global del programa didáctico de la materia por parte del profesor. El siguiente subapartado analiza cómo debe ser un buen podcast para el aprendizaje de una segunda lengua.

\subsection{Pautas de diseño técnico-instruccional para el profesor y de utilización por parte del estudiante}

Como hemos visto, los podcasts que se distribuyen por la red se pueden adquirir, en su mayoría, por medio de una suscripción gratuita. Una vez suscritos, los podcasts se envían 
de manera regular a través de Internet o de una red educativa y se puede acceder a ellos desde un dispositivo móvil. Dado que un podcast puede ofrecer cualquier tipo de contenido albergado en un archivo de audio o vídeo, podemos encontrar elementos potencialmente valiosos para el aprendizaje de segundas lenguas, por ejemplo, episodios de curiosidades científicas, ponencias grabadas o una demostración de principios de biología.

Las nuevas generaciones de estudiantes que ha crecido y vivido con la tecnología digital pueden utilizar podcasts de forma fácil y eficiente con sus dispositivos (tales como teléfonos celulares, MP3, MP4) para promover el aprendizaje móvil y enriquecer y acelerar su adquisición de destrezas conectándose en contextos reales y cambiantes, siguiendo la máxima de "a cualquier hora en cualquier sitio" (o mejor, como precisan Read \& Bárcena [2015] en alusión a la conveniencia de incorporar el contexto como una valiosa variable más del proceso de aprendizaje: "sometime, somewhere"). Así pues, se pueden emplear las grabaciones en cualquier momento y no limitarse a los tiempos de estudio fijos (Townend, 2005). Esto afecta la motivación de los estudiantes, disminuye el miedo a aprender, aumenta el tiempo de exposición y, por todo ello, es previsible que mejoren sus resultados.

En este contexto, los estudiantes también pueden crear y publicar materiales y mostrárselo a sus profesores o compañeros. Existe una amplia variedad de contenido educativo que se está desarrollando y divulgando en forma de podcast. Este contenido puede ser desde una presentación sobre un tema del plan de estudios hasta una comunidad de formación del profesorado en la que los docentes intercambian contenido y mejores prácticas con los miembros. Cualquier estudiante que tenga la posibilidad de "jugar a tener una radio virtual" obtiene en el proceso, periodístico y técnico, importantes habilidades cognitivas y sociales, ya que el hecho de reportar eventos, entrevistar personas y manejar la tecnología para digitalizar los contenidos y transmitirlos en Internet, involucra varias habilidades y destrezas altamente valoradas en el mundo globalizado en el que se desarrolla o desarrollará la vida laboral de los estudiantes. Además, en el caso de los estudiantes más jóvenes, es de adicional interés la orientación vocacional que puede significar la realización de un programa radial, ya que entrarán en contacto con las distintas actividades y profesiones vinculadas al medio de comunicación radial.

Veamos a continuación algunas pautas de diseño técnico-instruccional con podcasts por parte del profesor:

- La selección de los temas debe ser lo más específica posible. También se considera factible la creación de capítulos que introduzcan o den una idea global de un contenido más amplio.

- La integración de este tipo de material debería ser conceptualizada como parte del contenido de aprendizaje de la materia, no como un elemento adicional.

- Los elementos comunes a todos los capítulos deberían ser, por lo menos, tres: introducción y objetivos, contenidos y conclusión.

- El guión debe ser predominantemente pro-activo: resulta aconsejable ir realizando preguntas que lleven al alumno a pensar, para después optar por una respuesta o por un camino que lleve a encontrarla.

- En el caso de utilizar grabaciones ya existentes: música, grabaciones de personajes históricos, citas habladas etc., habrá que contemplar todo lo relativo a los derechos de autoría o reproducción (para ello es recomendable informarse en sitios oficiales como CEDRO). 
- Supone que el docente "se acerca" y orienta su metodología a esta nueva "generación digital" cuya relación cotidiana con la tecnología es muy diferente de las generaciones anteriores (Prensky, 2001).

Cabe destacar, entre estas pautas, la idea de que debe evitarse tanto el aislamiento y la desconexión de la actividad respecto al conjunto de la experiencia educativa, como la escucha pasiva del podcast. Bien al contrario, la actividad debe llevar al usuario a ser proactivo, por ejemplo, realizar una tarea, revisar un material, llevar a cabo una experiencia de campo, una auto-evaluación, un trabajo colaborativo, contraste de información, etc.

También pueden establecerse pautas de utilización por parte del estudiante, cuyo seguimiento ha de ser supervisado por el profesor, tales como la separación entre el uso del dispositivo móvil independientemente del habitual uso orientado al ocio que se suele hacer. Debe instársele también a que desarrolle su metacognición y su creatividad y pruebe a acceder y utilizar los materiales en distintos lugares y momentos, preferiblemente en sesiones cortas y frecuentes, y reflexione brevemente sobre lo satisfactoria que haya podido saber la experiencia y el aprovechamiento alcanzado, así como formas en que pueda mejorarse la sesión de aprendizaje realizada.

La utilización de formatos de audio añade un valor adicional a los habituales sistemas de aprendizaje de segundas lenguas (normalmente homogéneos en exceso). Este tipo de aprendizaje conlleva un intrincado entramado de competencias y procesos receptivos, productivos, interactivos, escritos y orales. Dada la ventaja que históricamente llevan los sistemas y herramientas escritos sobre los orales y la propia naturaleza de los podcasts, se explica la tendencia generalizada a verlos como valiosos recursos para la práctica y mejora de las destrezas orales.

\subsection{Los podcasts y el desarrollo de las destrezas orales}

La segunda generación de la Web, conocida mundialmente como Web 2.0, ha proporcionado nuevas formas de comunicación en línea (Warschauer \& Grimes, 2007), con sus herramientas exclusivas para la comunicación, la interacción y la colaboración (Bennett \& Gadlin, 2012). La Web 2.0 se concentra en las amplias aplicaciones como blogs, redes sociales y el podcasting, de modo que se puede invitar a los usuarios a la creación de contenidos y recursos web, en lugar de hacer simplemente un uso pasivo de ellos (Anderson, 2007). Debido a los beneficios educativos y las potencialidades de las tecnologías 2.0, éstas han cobrado enorme popularidad en todo el mundo y son utilizadas ampliamente tanto por los profesores como por los estudiantes (Santiago \& Navaridas, 2012).

Entre estas herramientas 2.0, muchos son los autores que han considerado que los podcasts pueden ser muy útiles para fines educativos, y en particular para las segundas lenguas (O’Bannon, Lubke, Beard \& Britt, 2011). Como ya hemos señalado anteriormente, pueden ser distribuidos en formato de audio o de video (conocidos entonces como vidcast), siendo estos archivos audiovisuales que se distribuyen en un formato digital a través de Internet y que son accedidos por parte de los estudiantes en sus ordenadores personales o dispositivos móviles (Kay \& Kletskin, 2012).

Los podcasts proporcionan contenido educativo en un formato apropiado para las necesidades formativas de los estudiantes en materia de segundas lenguas, que está no solo disponible cuando y donde quiera (Lazzari, 2009), sino que es susceptible de diversificarse 
y enriquecerse a medida que cambian los contextos de uso. El concepto de movilidad en el aprendizaje se considera como un valor añadido de primer orden en un entorno didáctico (Santiago, 2013). En el ámbito de las segundas lenguas, y en concreto de la comunicación oral, los podcasts se pueden utilizar para diferentes propósitos, como la sustitución parcial o total de clases en el aula, en las que la falta de elementos de estímulo y apoyo a la comunicación (objetos, temas, situaciones reales, etc.) y también la diversidad de input o modelos de referencia (a menudo solo el proveniente del propio profesor) suponen un grave hándicap para el desarrollo de destrezas que han de ser puestas en práctica, de forma universal, para participar en un número indeterminado de actos comunicativos con un número indeterminado de hablantes enormemente diversos entre sí (por su variante geográfica, nivel cultural, ocupación, edad, registro o sexo, entre otras variables).

Según Rosell-Aguilar (2007), podemos distinguir dos tipos de podcasts de uso frecuente en el contexto del aprendizaje de una segunda lengua:

- Podcasts 'auténticos', que tienen contenidos auténticos que son producidos y utilizados por los hablantes nativos como noticias.

- Podcasts 'cursos de idiomas', que pueden ser categorizados en elementos independientes y complementarios.

La investigación en este campo muestra la existencia de abundante evidencia de que los podcasts tienen multitud de beneficios educativos para la mejora de los distintos aspectos que conforman la competencia oral de una lengua, como la pronunciación, la entonación, la fluidez y las estrategias de cortesía conversacional, por nombrar algunos. Para ello en las clases de segundas lenguas se puede hacer uso de materiales auténticos disponibles para su descarga (Thorne \& Payne, 2005), combinar las ventajas de radio y otro tipo de reproductores (McLoughlin \& Lee, 2007) y aumentar la creatividad, el aprendizaje autónomo y la colaboración (Clothey \& Schmitt, 2008; Rahimi \& Katal, 2012). Los podcasts proporcionan una forma de trabajar las competencias orales en segundas lenguas de los estudiantes, bien sea a través del visionado o de la escucha - guiados, es decir, con pautas metodológicas, antes, durante y después de la exposición visual/auditiva - o dándoles oportunidades para crear y publicar sus propios materiales, teniendo como referente una audiencia real (Salmon \& Edirisingha, 2008), que se ha demostrado que mejora ostensiblemente la motivación intrínseca y extrínseca (Jenkins \& Lynch, 2006; O’Bryan \& Hegelheimer, 2007), no solo por la repercusión y visibilidad del trabajo, sino que por lo general se genera un clima de mejora en la relación de los estudiantes con el grupo y también en la retroalimentación personal (France \& Wheeler, 2007).

Entre los investigadores que han trabajado los podcasts para el desarrollo de las destrezas orales, Chan \& Lee (2005) parten de la reflexión de que el consumo y la producción de audio han sido muy descuidados y subutilizados en la enseñanza/aprendizaje de segundas lenguas en el pasado. Según estos autores, el uso de estas herramientas y recursos permiten un aprendizaje auténtico (si el emisor es un hablante de la lengua de destino y su fin ajeno al didáctico) y contextualizado (a diferencia de los ejercicios tradicionales de pronunciación simples, basados en la repetición de sonidos, palabras o breves frases aisladas a partir de un modelo). Estas propiedades de los podcasts permiten a los profesores crear tareas verdaderamente significativas para los estudiantes, que tienen mayores posibilidades de producir los esperados resultados de retención a largo plazo y reutilización creativa en nuevos escenarios comunicativos. 
McQuillan (2006a y b) describe varias tareas que se centran en la producción oral, tales como la creación de los diarios de audio, la realización de entrevistas con hablantes nativos y muestras de producción de los propios estudiantes que "pueden grabarse a sí mismos y a sus compañeros de clase y proporcionar muestras de voz al profesor para la evaluación". Tavales \& Skevoulis (2006) también sugieren que los estudiantes puedan grabarse a sí mismos y a hablantes nativos para luego participar en la práctica de escuchar y realizar comparaciones explícitas de la pronunciación, la gramática o la entonación. Estos análisis valorativos tienen un alto valor pedagógico tanto para el que recibe el feedback como para el que lo genera, ya que posibilitan un aprendizaje metacognitivo. En cuanto al tipo de soporte tecnológico, han llevado a cabo un estudio sobre el aprendizaje con iPods específicamente de la pronunciación y el vocabulario, frente al sistema "convencional" del aula 1.0 con materiales impresos. Aunque no se encontraron diferencias significativas en los grupos inmediatamente tras el estudio, después de 2 semanas los participantes del grupo experimental demostraron haber retenido el significado del $40 \%$ de nuevas palabras a las que habían sido expuestos, mientras que sólo el $27 \%$ había sido retenido por el grupo de control.

En un estudio de Lord (2008) sobre pronunciación con un podcast desarrollado ex profeso para la enseñanza de lenguas extranjeras, diecinueve estudiantes en una clase de fonética trabajaron con trabalenguas, lecturas cortas y reflexiones personales sobre su propia pronunciación. Lord empleó el popular Cuestionario de Actitudes para la Pronunciación (Elliott, 1995), así como las puntuaciones de seis tareas orales, calificadas por tres jueces sobre la capacidad global para la pronunciación. Tanto las actitudes como las habilidades de pronunciación se evaluaron pre-semestre y post-semestre. Hubo una apreciación unánime de progreso en ambos casos, razón por la que se mantuvieron a disposición de los estudiantes una vez finalizado el proyecto, con el fin de que los estudiantes pudieran seguir trabajando la mejora de la pronunciación en el futuro.

En la última década han proliferado experiencias similares con resultados positivos. También desde la propia comunidad de profesores y de estudiantes se han hecho públicos listados comentados de podcasts útiles para el desarrollo de las destrezas orales en segundas lenguas (por ejemplo, http://www.languagesurfer.com/2014/07/14/10-best-podcasts-for-language-learning/ y http://www.theguardian.com/education/2015/feb/09/top-podcasts-learninglanguage-german-japanese). Aparte de la movilidad y la gratuidad, se valora la verbalidad que es objeto de estudio dentro del vasto y complejo escenario de las segundas lenguas, como observa Gullekson (2014): "With podcasts, you'll hear proper pronunciation, you'll improve your listening comprehension, and you'll get all the benefits that come with that". Por último, son de particular interés las experiencias didácticas en las que se extienden los límites del aula ofreciendo a los estudiantes la posibilidad de que creen y publiquen su propio contenido para audiencias reales. Se observa que de esta forma los estudiantes se responsabilizan más de su trabajo, prestan más atención y realizan un esfuerzo mayor ante la perspectiva de una repercusión tan dilatada.

\section{Conclusión}

Este artículo ha analizado el podcasting como una de las tecnologías digitales nacidas en este siglo que ha recibido una progresiva acogida en entornos no necesariamente relacionados 
con el mundo informativo en el que surgió. Como se ha explicado, en el de la educación, y en concreto, en el ámbito de la didáctica de las segundas lenguas, pronto se ha encontrado una aplicabilidad para los podcasts, no solo por sus propias características sino por las de su habitual soporte tecnológico, interconectado y móvil.

Por último, cabe destacar la flexibilidad del podcasting en manos del profesor y la diversidad de enfoques metodológicos que pueden adoptarse, tanto en enseñanza presencial, a distancia, híbrida o flipped, como se ha mencionado en el artículo. Dado el avance paulatino que está experimentando la sociedad - y, por lo tanto, la educación - hacia un modelo 3.0 de apertura, interconexión y compartición sin precedentes, los autores de este artículo prevén en los próximos años la proliferación de proyectos colaborativos de intercambio, elaboración conjunto o explotación didáctica de podcasts entre estudiantes de otras partes del mundo, integrados en modalidades didácticas revolucionarias recientes que también tienen margen de desarrollo como son los los LMOOC (language MOOCs o cursos en línea abiertos y masivos de lenguas; Martín \& Bárcena, 2014) y el aprendizaje personalizado (en el que son los estudiantes los que llevan la iniciativa de sus propios diseños didácticos; Fullan, 2009), que permitirán explorar el podcasting en combinación con otras propuestas innovadoras como solución a los grandes retos educativos del siglo XXI.

\section{Bibliografía}

Anderson, P. (2007). What is Web 2.0? Ideas, technologies and implications for education. JICS Technologies and Standards Watch. Disponible en: http://www.ictliteracy.info/rf.pdf/ Web2.0_research.pdf, consultado 1 marzo 2016.

Bennett, L.M. \& Gadlin, H. (2012). "Collaboration and team science: from theory to practice". J. Investig. Med. 60(5): 768-75. Disponible en: http://www.ncbi.nlm.nih.gov/pmc/articles/ PMC3652225/, consultado 1 marzo, 2016.

Chan, A. \& Lee, M.J.W. (2005). "An MP3 a day keeps the worries away: Exploring the use of podcasting to address preconceptions and alleviate pre-class anxiety amongst undergraduate information technology students". En D.H.R. Spennemann \& L. Burr (Ed.), Good Practice in Practice: Proceedings of the Student Experience Conference, Wagga Wagga, NSW: 58-70.

Clothey, R. \& Schmitt, C. (2008). "Education in Motion: Innovating with iPods". In J. Luca \& E. Weippl (Ed.), Proceedings of EdMedia: World Conference on Educational Media and Technology 2008. Association for the Advancement of Computing in Education (AACE), 629-639. Disponible en: https://www.learntechlib.org/p/28458, consultado 1 marzo, 2016.

Elliott, A.R. (1995). Foreign language phonology: Field independence, attitude, and the success of formal instruction in Spanish pronunciation. The Modern Language Journal, 79 (4): 530.

France, D. \& Wheeler, A. (2007). Reflections on using podcasting for student feedback Planet, 18: 9-12.

Fullan, M. (2009). Michael Fullan's Answer to "What is Personalized Learning?" Microsoft Partner Network. Disponible en: http://cs.mseducommunity.com/wikis.personal.michaelfullan-s-answer-to-quot-what-is-personalized-learning-quot/revision/3.aspx, consultado 1 marzo, 2016.

Gullekson, R. (2014). Language Master Key: How to Unlock Your Brain's Ability to Learn Any Language. Cypress Dome Publishers. 
Jenkins, M. \& Lynch, K. (2006). I want to tell you a story... Proceedings of the 23rd annual ASCILITE Conference, Who's Learning? Whose Technology. University of Sidney, Australia.

Kay, R. \& Kletskin, I. (2012), Evaluating the use of problem-based video podcasts to teach mathematics in higher education, Computers and Education, 59: 619-627.

Lazzari, M. (2009) Creative use of podcasting in higher education and its effect on competitive agency, Computers and Education, 52(1): 27-34.

López Vidales, N. \& González Aldea, P. (2014). Audioblogs y TVblogs, herramientas para el aprendizaje colaborativo en Periodismo. Disponible en: http://www.ocendi.com/descargas/ comunicar_42.pdf, consultado 1 marzo, 2016.

Lord, G. (2008). Podcasting communities and second language production. Foreign Language Annals, 41: 364-379.

McLoughlin, C. \& Lee, M.J.W. (2007). Social software and participatory learning: Pedagogical choices with technology affordances in the Web 2.0 era. In ICT: Providing choices for learners and learning. Proceedings of ASCILITE Singapore 2007. Disponible en: http:// www.ascilite.org.au/conferences/singapore07/procs/mcloughlin.pdf, consultado 1 marzo, 2016.

McQuillan, J. (2006a). Languages on the go: Tuning in to podcasting. The International Journal of Foreign Language Teaching, 2(1): 16-18. Disponible en: http://www.tprstories.com/ijflt, consultado 1 marzo 2016.

McQuillan, J. (2006b). iPod in education: The potential for language acquisition. (libro blanco). Disponible en: http://edcommunity.apple.com/ali/galleryfiles/12071/iPod_Edu_Whitepaper_Language_Aquisit ion.pdf, consultado 1 marzo, 2016.

O’Bannon, B., Lubke, J., Beard, J. \& Britt, G. (2011). Using Podcasts to Replace Lecture: Effects on Student Achievement. In M. Koehler \& P. Mishra (Ed.), Proceedings of Society for Information Technology \& Teacher Education International Conference 2011. Chesapeake, VA: Association for the Advancement of Computing in Education (AACE): 2616-2623. Disponible en: https://www.learntechlib.org/p/36708, consultado 1 marzo, 2016.

O'Bryan, A., \& Hegelheimer, V. (2007). Integrating CALL into the classroom: The role of podcasting in an ESL listening strategies course. ReCALL, 19(2): 162-180. Disponible en: http://dx.doi.org/10.1017/S0958344007000523, consultado 1 marzo, 2016.

Prensky, M. (2001). Digital Natives, Digital Immigrants. On the Horizon, 9(5), MCB University Press.

Rahimi, M. \& Katal, M. (2012). The role of metacognitive listening strategies awareness and podcast-use readiness in using podcasting for learning English as a foreign language. Computers in Human Behavior, 28(4): 1153-1161.

Read, T. \& Bárcena, E. (2015). Toward mobile assisted Language MOOCs. En A. Mesquita \& Paula Peres (Ed.) Furthering Higher Education Possibilities through Massive Open Online Courses, Nueva York: IGI Global: 225-244.

Rosell-Aguilar, F. (2007). Top of the pods - In search of a podcasting "Podagogy" for language learning. Computer Assisted Language Learning, 20: 471-492.

Salmon, G., Edirisingha, P. (ed.) (2008) Podcasting for Learning in Universities, Maidenhead, Reino Unido: Open University Press, McGraw-Hill. Disponible en: http://www2.le.ac.uk/ search?SearchableText=podcast, consultado 1 marzo, 2016.

Santiago, R. (2013) Tecnología móvil e innovación en el aula: nuevos retos y realidades educativas. En R.M. Goig Martínez (ed.). Formación del Profesorado en la Sociedad Digital. Investigación, Innovación y Recursos Didácticos. Madrid: Editorial UNED.

Santiago, R. \& Navaridas, F. (2012) La Web 2.0 en escena. Web 2.0 comes on the scene. PixelBit. Revista de Medios y Educación, 41: 19-30. 
Tavales, S., \& Skevoulis, S. (2006). Podcasts: Changing the face of e-learning. Disponible en: http://ww1.ucmss.com/books/LFS/CSREA2006/SER4351.pdf, consultado 1 marzo, 2016.

Thorne, S.L., \& Payne, J.S. (2005). Evolutionary Trajectories, Internet-mediated Expression, and Language Education. CALICO Journal, 22(3): 371-397.

Tourón, J., Santiago, R. y Díez, A. (2014). The Flipped Classroom: Cómo convertir la escuela en un espacio de aprendizaje (Innovación educativa). Digital-Text.

Townend, N. (2005). Podcasting in higher education. Viewfinder, Media Online Focus, 61, British Universities Film \& Video Council: i-iv.

Veiga Marriott, R. \& Lupion Torres, P. (2009). Handbook of Research on eLearning Methodologies for Language Acquisition. Information Science Reference, Nueva York: IGI Global.

Warschauer, M. \& Grimes, D. (2007). Audience, authorship, and artifact: the emergent semiotics of Web 2.0. Annual Review of Applied Linguistics, 27: 1-23. Boston: Cambridge University Press. 